\title{
La responsabilidad social corporativa en modo sistémico: dos modelos alternativos
}

\author{
Corporate Social Responsibility in a Systemic Way \\ Two Alternative Models
}

\section{Responsabilidade social corporativa de forma sistêmica: dois modelos alternativos}

\author{
Alejandra Raza \\ Pontificia Universidad Católica del Ecuador (Quito, Ecuador) \\ alee.razam@gmail.com
}

DOI: https://doi.org/10.32719/25506641.2018.4.4

Fecha de presentación: 16 de abril de 2018 • Fecha de aceptación: 26 de septiembre de 2018 Artículo de investigación

Licencia Creative Commons

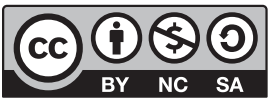




\section{Resumen}

La visión de la responsabilidad social ha generado controversia en el mundo empresarial y, finalmente, se ha minimizado su importancia. El entorno en el que se encuentra inmersa una organización sufre los impactos generados por las actividades empresariales cotidianas que deben ser resueltos de una u otra manera; sin embargo, la función sistémica del mercado ejerce influencia en la definición de cuál es la mejor manera de mitigar o evitar tales impactos que pudieran generar una problemática social. En este artículo se argumenta que la teoría de sistemas clásica y la contemporánea proponen dos perspectivas que generan modelos de RSC totalmente diferentes pero aplicables a la gestión para el manejo de estos impactos.

Palabras clave: Responsabilidad social corporativa, teoría de sistemas, internalización, externalidades, regulación.

JEL: M14 Cultura corporativa, responsabilidad social corporativa.

\section{Abstract}

There has been controversy around the idea of social responsibility in the business sector, nevertheless its importance has been rarely acknowledged. The daily economic operations of an organization have negative effects upon its environment that must be dealt with in some way or another; yet, the systemic economic logic of the organization normally defines the ways to cope with social problems in terms of market mechanisms. This article contends that classic and contemporary systems theory offer two different and totally opposed models of social corporative responsibility that can be applied to manage these negative effects.

Keywords: Corporative social responsibility, systems theory, internalization, externalities, regulation.

JEL: M14 Corporate culture, corporate social responsibility.

\section{Resumo}

A visão de responsabilidade social gerou polémica no mundo dos negocios e, por fim, sua importancia foi minimizada. $\mathrm{O}$ ambiente em que uma organização está imersa sofre os impactos gerados pelas atividades diárias do negócio que deven ser resolvidos de uma forma ou de outra; no entanto, a função sistémica do mercado influencia a definição da melhor maneira de mitigar ou evitar tais impactos que poderiam gerar um problema social. Neste artigo, argumentase que a teoría clássica e contemporãnea dos sstemas propôe duas perspectivas que geram modelos de RSC completamente diferentes, mas aplicáveis à gestão para o gerenciamiento desses impactos.

Palavras-chave: Responsabilidade social coporativa, Teoria dos sistemas, Internalização, Externalidades, Regulação.

JEL: M14 Cultura corporativa, responsabilidade social corporativa. 


\section{Introducción}

L

a responsabilidad social corporativa (RSC), constituye una visión ética -que suele ser contraria a la visión capitalista de acumulación inherente a los negocios- que resulta un imperativo en el mundo contemporáneo por los efectos negativos que tienen algunas actividades en los stakeholders y en la sociedad en general. Aunque el tema de responsabilidad social ha sido estudiado durante muchos años, no suele ser implementado de la manera adecuada -en términos de voluntariedad- en las empresas (Porter y Kramer 2006). La responsabilidad social se traduce como el manejo de los impactos negativos causados por grandes empresas en el medioambiente, en la sociedad, la economía, la política y en los propios colaboradores de la empresa; este enfoque se ha visto limitado por los dirigentes de algunas compañías cuya pretensión es exclusivamente empresarial. Se podrían mencionar muchos ejemplos en los que se visualiza claramente la poca importancia que se le reconoce al bienestar de los diferentes actores del entorno de la empresa, mientras que lo contrario sucede con la preocupación respecto al incremento de la utilidad y eficacia.

La idea de RSC nació en la década del 50 y ha venido tomando forma hasta la actualidad con la aparición de instituciones dedicadas exclusivamente a la aplicación de esta teoría; sin embargo, con el pasar del tiempo, los cambios vertiginosos de los últimos veinticinco años, fundamentalmente derivados de la globalización, determinaron modificaciones en el enfoque de la responsabilidad social (Jaramillo 2007) que deben ser tomadas en cuenta en cualquier sector económico y a todo nivel empresarial.

Desde la aparición de la RSC se han generado diferentes iniciativas al interior de gremios profesionales, agencias acreditadoras, organismos multilaterales y también en las esferas gubernamentales, respecto a la pertinencia de la aplicación de diversas políticas, con el propósito de lograr eficacia en 
la implementación de este modelo en las organizaciones; el Pacto Mundial (2000) implementado por las Naciones Unidas, la Declaración Tripartita de la OIT sobre empresas internacionales y política social (1997-2000), la norma AA1000 realizada en 1999 por el Institute of Social Ethical Accountability y la SA8000 promovida por la agencia acreditadora del Council of Economic Priorities (De la Cuesta y Valor 2003) son evidencias de estas iniciativas. La implementación de la RSC, estrictamente desde la perspectiva económica neoliberal, supone que las distintas organizaciones tienen como objetivo único la maximización de utilidades -visión capitalista- y que no tienen la obligación de responder ante la sociedad, pues esa responsabilidad es de una entidad mayor: el Estado (Carroll y Shabana 2010). Desde una visión social, la RSC considera que al empresario le conviene ser responsable socialmente (Fernández 2016) para que su actividad sea sostenible a largo plazo; otros aspectos a referir en esta perspectiva son la capacidad y habilidad que teóricamente tienen las organizaciones de solucionar problemas sociales y la aseveración de que es mejor ser proactivo que reactivo. El ser proactivo se relaciona directamente con la anticipación y planificación; mientras que ser reactivo es accionar solamente cuando ya se han generado condiciones que han derivado o pueden derivar en situaciones de problemática social, en cuyo caso la intervención de la empresa suele resultar más costosa (Carroll y Shabana 2010).

De otra parte, debe considerarse que las empresas están inmersas en un contexto mayor que su propio espacio. En efecto, la empresa debe su permanencia y crecimiento a la comunidad y a las partes involucradas con su gestión, por lo que su actividad debe ser tomada en cuenta con un enfoque sistémico. En esta perspectiva, debe relacionarse la responsabilidad social corporativa con la teoría de sistemas, y con este propósito, es pertinente evaluar la concepción clásica de esta teoría, seguido de la teoría contemporánea, para establecer el mejor enfoque de lo que debe ser la RSC en la administración de empresas.

Según lo previamente expuesto, el objetivo de este estudio es identificar las características de los dos enfoques, el clásico y el contemporáneo, de la teoría de sistemas y proponer la mejor perspectiva para aplicar un modelo de responsabilidad social corporativa en la gestión. El método utilizado para alcanzar este objetivo fue la investigación bibliográfica respecto al marco teó- 
rico y a los estudios temáticos desarrollados en los últimos años. En cuanto al marco teórico, el estudio se sustenta en la teoría de sistemas clásica de Ludwig Von Bertalanffy y en la perspectiva contemporánea de Niklas Luhmann; y con relación a los estudios temáticos, se ha explorado la correspondiente información en las bases bibliográficas.

\section{RSC desde la teoría de sistemas clásica: responsabilidad como internalización de las externalidades}

La teoría clásica de sistemas define a los sistemas como un conjunto de elementos interrelacionados e interdependientes entre sí -que están compuestos a su vez por subsistemas- y que tienen un fin común. Esta teoría revela el funcionamiento holístico de diferentes unidades compuestas por diversos factores y clasifica a las ciencias y elementos en diferentes tipos según su desarrollo y sus objetivos; afirma, además, que los diferentes elementos conforman un sistema unitario, es decir, no pueden comprenderse individualmente, sino en un conjunto que determine sus propiedades (Von Bertalanffy 1976a).

En el caso empresarial, y dentro de esta perspectiva, la teoría de sistemas define a las organizaciones como sistemas abiertos compuestos por lo general de diferentes subsistemas -áreas de una empresa- cuyo funcionamiento viene determinado por el objetivo empresarial fundamental, a saber: la generación de utilidad. Los resultados que obtiene una empresa se producen por una transacción o interacción con el ambiente en el que esta desarrolla su actividad y con los diferentes interesados. En otras palabras, las empresas tienen influencia del entorno y de todos sus actores. En realidad, estos impactos tienen una consecuencia, que puede ser negativa o positiva, sobre el entorno. La generación de riqueza requiere la utilización de factores del entorno -input o insumo energético del sistema- los que se procesan de manera que se obtiene rentabilidad - output o resultado energético- (Von Bertalanffy 1976b) sin tomar en cuenta las repercusiones que podrían generar en el entorno. Las empresas que optan por un modelo de RSC deben enfrentar el dilema de tomar acción ante efectos, cuyo objetivo central no se relaciona 
con la generación de utilidad en las empresas y por el contrario se genera un incremento en el costo empresarial.

Para que la RSC tenga mayor acogida en las operaciones cotidianas de grandes compañías, se han hecho investigaciones, como el Corporate Social Performance de Burke y Logsdon en 1996, que revelan que la rentabilidad en términos de creación de valor y mejora en la competitividad, va de la mano con la responsabilidad social - por temas de marketing-(Toro 2006). Hoy en día se puede observar la tendencia que tienen los consumidores de elegir empresas que manejan modelos de ética empresarial hacia la sociedad, creando así valor para las compañías -en términos de rentabilidad y de competenciay para actores del entorno. Este es el caso de TOMS shoes, que maneja un modelo de One for One con el cual trata de ayudar a personas necesitadas, por la compra de cualquier producto. ${ }^{1}$ Este es el objetivo central que tiene la responsabilidad social corporativa: el valor compartido; empero, persisten creencias opuestas que consideran que los problemas que enfrentan las partes relacionadas son ajenos a la empresa y por ello se ha dejado de lado o se ha minimizado la responsabilidad social corporativa.

El valor compartido asume que todo proyecto u operación de una compañía generará beneficios para todas las partes interesadas o involucradas. La filosofía de ganar-ganar se genera en oposición al imperativo del capitalismo (Fleming, Roberts y Garsten 2013). El capitalismo es generalmente la ideología base para la producción y por ende para las corporaciones, sin importar su tamaño o actividad, por lo que se genera conmoción al relacionar estos dos modelos - capitalismo y RSC- como fundamento para el desarrollo de actividades; empero hay que tener presente que en el mundo contemporáneo las organizaciones deben tener una capacidad de aprendizaje y reflexividad para poder subsistir en un entorno complejo, manejando sus propios problemas (Mascareño y Drago 2016). En realidad, algunos estudios, como el

1. El modelo One for One de TOMS inició de manera no intencional; sin embargo, ha generado un impacto tan grande en el mercado que la empresa ahora tiene la posibilidad de ayudar a personas que viven en extrema pobreza no solo con zapatos -que es uno de los productos de la compañía- sino también con salud, agua potable, partos seguros y prevención de bullying; por ello, TOMS y su fundador recibieron un premio reconocido a nivel global. La empresa ha crecido en el mercado con las prácticas aplicadas de RSC. En una entrevista el fundador de TOMS dijo que esta iniciativa ha sustituido los costos que se emplean en marketing y publicidad, lo que hace que sea aún más rentable este modelo (TOMS 2006). 
Carbon Trust, afirman que las empresas que se enfrentan al cambio climático han tenido la oportunidad de aumentar el valor de la empresa hasta en un 80\% (Agudelo 2013).

Se puede explicar de mejor manera que lo que genera el problema central de establecer una posible relación entre un modelo de RSC y la teoría de sistemas es la falsa percepción que los empresarios tienen de cuáles son los elementos que conforman la empresa, debido a que se toman en consideración únicamente los componentes del microambiente o internos de la compañía y las partes interesadas y se deja de lado a los actores que pueden estar involucrados en las operaciones, así sea de manera involuntaria (De la Cuesta y Valor 2003).

Empero, hay que tener presente que existen programas de RSC que han generado un gran impacto a nivel de su microentorno que cabría mencionar en el ensayo, como es el caso ecuatoriano de Tonicorp. ${ }^{2}$ Esta compañía maneja una cadena de valor compartido, lo que se traduce en la creación de valor en toda la cadena de suministros: desde los clientes, a la comunidad e incluso al medioambiente. El beneficio de la comunidad se basa en la protección del entorno y la salud fortaleciendo la alimentación saludable, la educación y la concientización ambiental. Por otro lado, han desarrollado el programa Yo soy ganadero, el que promueve el desarrollo sustentable de la ganadería utilizando tres sistemas integrales.

En base a este ejemplo, cabe afirmar que la responsabilidad social corporativa no debe limitarse a ser instrumental: debe tener una mayor perspectiva en la que se refleje a las compañías como parte de un conjunto o sociedad mucho más grande y que se toman en cuenta todos los stakeholders, incluyendo las partes involucradas involuntariamente (Kamppinen, Vihervaara, y Aarras 2008). Es decir, para la máxima comprensión de la RSC, es necesario tener en cuenta a los factores externos de una compañía, que son frecuentemente ignorados en el momento de solucionar problemas. De esta manera se alcanzaría un bienestar global que abarque, en este caso, a todos los subsistemas para una correcta implementación de un programa, modelo de RSC. Por lo tanto, para evitar prácticas de outsoursing y poder tener una

2. Tonicorp mantiene la filosofía de que para mantener éxito empresarial en el largo plazo, se debe mantener relaciones estrechas con el desarrollo y bienestar que pueden generar como empresa en las comunidades donde operan (Industrias Lácteos 2018). 
mejor comprensión del funcionamiento de una empresa en contexto macro, el modelo de responsabilidad social pretende manejar la relación sistémica que mantiene la compañía con sus stakeholders. Esta aseveración se refiere a la capacidad que tiene una compañía de internalizar las externalidades, siempre y cuando lo hagan en base a la lógica interna de la compañía o, en otras palabras, bajo sus propios códigos ligados a la monetización y a la generación de utilidad.

Las externalidades son impactos que genera un actor económico, que sufren otros y que no son resueltas por quien los originó. En el momento en que se internalizan estos impactos, cada actor económico se encarga de los costos de los que es responsable o, mejor dicho, se hace cargo de los impactos causados a terceros por actividades que las beneficiaron únicamente a ellos (Godwin 2007). Un claro ejemplo de este proceso es el mercado de carbono, que es considerado un esfuerzo para mitigar el cambio climático en conjunto con la reducción de emisiones de gases que producen el efecto invernadero en países muy industrializados. Para ello se han implementado mecanismos de mercado que se encargan básicamente de efectivizar transacciones de compra-venta de emisiones de gases con efecto invernadero entre países, empresas o incluso individuos. Para mantener una normativa de este tipo de transacciones, el mercado regulado se basa en a) proyectos registrados y verificados y, b) la fijación de niveles máximos de producción de gases, que de cierta manera obligan a las empresas a realizar transacciones para cumplir con la normativa (Carbon Trust 2018).

En el ejemplo anterior se revela la limitación de la visión empresarial contemporánea, pues se enfoca exclusivamente en la monetización de los efectos causados en terceros, en el corto plazo. Este tipo de interiorización mantiene a flote la ideología capitalista, sin entender que el manejo de un modelo de RSC puede traer ventajas competitivas a largo plazo, entre las que destaca la reducción de costos, la facilidad y factibilidad de procesos, aumento en la productividad, mayor fidelización del cliente, generación de nuevas alianzas, entre otros (Marín y Rubio 2008).

El caso del Grupo Éxito en Colombia es contrario al referido en el párrafo anterior. Esta empresa se propuso eliminar el uso de plásticos en el despacho y venta de sus productos utilizándose en cambio bolsas reutilizables, lo que estaba contemplado que forme parte de un plan de fidelización, el cual 
ha venido mejorando sus resultados con el pasar del tiempo. Esto refleja el manejo de un modelo de la RSC en el que aunque resulte más costoso, genera más beneficios a largo plazo obteniendo como resultado la sostenibilidad (De la Cuesta y Valor 2003).

\section{RSC desde la teoría de sistemas contemporánea: la tipificación legal que limita la reproducción de la lógica sistémica}

La teoría contemporánea, por su parte, se refiere a los grandes sistemas como complejos por la gran cantidad de elementos que posee; esto produce que en la toma de decisiones se considere los aspectos internos únicamente, dejando de lado elementos del entorno, porque no se los considera de gran importancia (Luhmann y Rodríguez Mansilla 1997). Por ende, se los minimiza o ignora cuando existen impactos negativos. Desde la perspectiva luhmanniana, los sistemas corren el riesgo de ser insostenibles si el medioambiente permite a las compañías tener su completa independencia (Valentinov 2013). Sin embargo, en el caso de las empresas hay que resaltar que los stakeholders primarios y secundarios afectan y se ven afectados por actividades de la compañía 18/01/2019 11:38:00 por lo que podrían inducir al cierre prematuro de la misma cuando la relación sistémica no satisfaga sus necesidades. A esto se suma la interconectividad en la que vivimos inmersos -la globalización- que fomenta la creación de nuevas redes de stakeholders a nivel mundial, lo que genera a su vez mayor presión en las compañías (Teubner 2012).

Por otro lado, cabe resaltar que la actividad que desarrolla cada empresa está siempre sujeta a lo dictado por el marco jurídico (Valencia, Escobar y Calderón 2017). En realidad, la perspectiva empresarial sugiere que generar utilidad está únicamente ligado a las limitaciones que presenta el Estado, es decir, que el cumplimiento de normas y leyes es el único compromiso que tienen las empresas con la sociedad dejando nuevamente de lado posibles efectos en algunos involucrados -los derechos humanos y la protección al medioambiente, por ejemplo-. En teoría, el Estado es el encargado de establecer condiciones para regular el mercado de manera que no se generen impactos negativos en el entorno, lo que implica que el modelo de RSC se 
vea más comprometido y menos aceptado por diferentes empresarios, pues se considera que el invertir en el bienestar social -y de los stakeholders-es obligación netamente del Estado y por ende no tiene ninguna relación con la actividad empresarial (Carroll y Shabana 2010).

Es globalmente conocido que hay entidades en diferentes partes del mundo y algunas otras que funcionan únicamente en su país de origen -como es el caso del CERES en el Ecuador-, que han fomentado la implementación del modelo de RSC en las compañías; sin embargo, existen algunas normas referentes a la responsabilidad social que ya son de carácter obligatorio por establecimiento estatal y son diferentes en cada país, como las obligaciones de cumplimiento ambiental, de higiene alimenticia y de seguridad industrial. Esto genera una gran inquietud en el mundo de los negocios, pues teóricamente la RSC es voluntaria. Si la RSC es voluntaria, al momento de regular el modelo, ¿se vuelve obligatoria? Y al ser obligatoria, obviamente pierde el sentido de responsabilidad empresarial, por el desinterés que se desarrollaría en las empresas para con la sociedad y los involucrados; al tener una perspectiva de la RSC equivocada, en referencia a la voluntariedad y obligatoriedad, se la vería simplemente como el cumplimiento de una exigencia legal. Estrictamente, la responsabilidad social corporativa intenta superar aquello que la empresa debe hacer por obligación legal, lo que quiere decir que el cumplir la ley no hace a una empresa socialmente responsable (Vera y Hernández 2017).

Lo anterior sugiere que no se debe sustituir un modelo legislativo que se enfoque en el bienestar social por la RSC, sino que deben ser complementarias entre sí; además, como las leyes pueden variar con el pasar del tiempo, es muy complejo mantener un modelo de RSC sostenible, lo que comprometería los resultados de la actividad empresarial.

De otra parte y en este contexto cabe referir las certificaciones ${ }^{3}$ reconocidas a nivel mundial que suelen ser adoptadas por algunas empresas y que ge-

3. La ISO 26000 permite que la RSC sea más comprensible en el mundo empresarial; pone a disposición conocimiento de desarrollo basado en consensos y en estándares relevantes de mercado internacional que apoya la innovación y brinda soluciones a cambios globales. La ISO tiene más de 22.308 estándares internacionales y documentos relacionados para casi todas las industrias, desde tecnología a higiene alimenticia, agricultura a salud. Respecto a la RSC se mencionan temas como derechos humanos, prácticas laborales, medioambiente, prácticas 
neralmente intentan mejorar la imagen de la compañía, lo que significa que pasan a ser de una norma a un estándar (Gutiérrez y González 2011) y que se constituye en una ventaja competitiva que debe ser afrontada por diferentes empresas para mantenerse en el mercado. Sin embargo, estas certificaciones son más guías que normas y tienen como objetivo el desarrollo sostenible.

No cabe duda de que el modelo de RSC genera controversia en los empresarios, pues implica cambiar su planificación institucional, su filosofía -visión, misión y objetivos- sus políticas y valores y su estructura organizacional, por un enfoque de valor compartido, lo que, además, debe verse reflejado en la toma de decisiones, en las actividades cotidianas y en nuevos proyectos. Por ello, se propone que la única manera de manejar la irresponsabilidad empresarial es por medio de regulación estatal (Sabogal 2008). De esta manera se crearía una normativa que se enfoque directamente en la $\mathrm{RSC}$, lo que generaría un vínculo indirecto entre procesos propios de la empresa y propios del macroentorno (Teubner 2012).

Para poder ejemplificar la eficacia de la limitación estatal, se ha tomado como referencia a la crisis hipotecaria del 2008 en Estados Unidos, la que fue provocada por la falta de regulación comercial (Gutiérrez y González 2011). Este motivo, entre otras posibles fallas de mercado -como la competencia perfecta, mercados abiertos, etc.- son razones por las que el Estado debe intervenir con normativas que regulen el mercado. Tomado este ejemplo, queda sobreentendido que, para poder aplicar este modelo de manera exitosa, los sistemas deberán reducir su nivel de libertad (Valentinov 2013) ¿A qué se refiere con reducir su nivel de libertad? A mantener el nivel de voluntariedad en conjunto con el cumplimiento de leyes o normas en lo que respecta a la RSC. De esta manera, no solo se mitigan posibles efectos negativos que se generan por determinadas actividades de una organización, sino que se evita a toda costa un posible impacto (Valentinov 2013), para lo cual, es útil estrechar lazos con los stakeholders, lo que significa hacer alianzas estratégicas cuyos resultados sean igual de favorables para ambos lados.

justas de operación, asuntos de consumidores, gobernanza organizacional y la participación y el desarrollo de la comunidad (ISO s. f.). La metodología del Global Report Initiative (GRI), por su parte, permite que los gobiernos y los negocios a nivel mundial comuniquen su impacto en temas de sustentabilidad como el cambio climático, los derechos humanos, gobernanza y bienestar social (Global Reporting Initiative s. f.). 


\section{Responsabilidad social corporativa: ¿administración de los efectos negativos o tipificación legal para evitar efectos negativos?}

En la actualidad, es común que la gente de negocios piense que el crecimiento de las compañías va de la mano con las relaciones estables -y beneficiosas- que esta establezca con todos los actores de su entorno (Jaramillo 2007); dicho de otras palabras, es importante que el valor que se cree por actividades empresariales privadas, sea compartido, lo que a su vez quiere decir que se debe dejar de lado el objetivo empresarial capitalista que es netamente individualista y se debe tener presente que las empresas son y funcionan como un sistema (Golob y otros 2014). Así, incluso se aumenta la posibilidad de alcanzar un desarrollo sostenible a largo plazo, por aplicar RSC dentro del ámbito en el que la empresa desarrolla su actividad. Sin embargo, la complicación nace de la pregunta ¿es necesario que una entidad superior regule los diferentes modelos de RSC? La lógica nos lleva a la respuesta más clara: el regular este modelo implica que la RSC no sea más que un cumplimiento de leyes estatales, alejándose así de lo que implica ser socialmente responsable, por su traducción en voluntariedad. En la actualidad, existen varias regulaciones impuestas por el Estado en este sentido; sin embargo, varían según el país y la actividad de la empresa. Además, se debe tener presente que la ética empresarial juega un papel muy importante para poder definir hasta qué punto es necesario tomar acción sobre los impactos causados a los stakeholders por la organización.

La RSC ligada con la teoría de sistemas, ha generado en muchos casos una respuesta incongruente a lo que se esperaría como estrategia o modelo de responsabilidad social, ya que se limita a ser socialmente responsable de manera lucrativa, es decir generar beneficios únicamente para la compañía sin considerar el impacto que tienen en los diferentes involucrados. Pero la RSC va más allá que eso, como se mencionó anteriormente: se debe tener presente que la aplicación de un modelo de este tipo, implica la generación de valor agregado a largo plazo y de ventajas competitivas duraderas (Gutiérrez y González 2011). A modo de ejemplo: si existe regulación por parte del Estado, se pueden formar nuevas alianzas estratégicas entre gobiernos y empresas privadas, de manera que estas últimas podrían tener un rol en la me- 
jora de problemas sociales de mayor magnitud -como en la Europa actual-. ${ }^{4}$ Esto evidencia que las empresas logran regularse a nivel social, económico y medioambiental, porque cuentan con un mayor nivel de responsabilidad para a su vez, ayudar a resolver problemas políticos en conjunto con actores del Estado y actores de la sociedad civil.

El internalizar externalidades de manera coherente -0 administrar los impactos creados-significa asumir de una manera directa la responsabilidad de los efectos por las diferentes decisiones tomadas para alcanzar los objetivos establecidos por una compañía; sin embargo, el proponer un modelo de este estilo significa asumir el correspondiente costo para la RSC junto a un modelo ético y moral de gestión que puede significar la reducción de costo en otras actividades o áreas como el marketing y la publicidad, como se expresó anteriormente en el caso TOMS. En cambio, al regular ciertas normas de responsabilidad social, se pretende asegurar un bienestar al entorno de manera absoluta -quitando el valor de voluntariado a la responsabilidad-e impulsar de cierta manera la implementación de modelos de moral y ética en las empresas.

En realidad, la idea de regular el modelo de RSC mantendría el mercado ordenado por algún tiempo; sin embargo, las compañías con visión hipercapitalista han dejado claro que son capaces de poner en riesgo cualquier $\operatorname{cosa}^{5}$-sea la sociedad, los trabajadores o el medioambiente- con el fin de cumplir su objetivo de rentabilidad, por lo que no sería de sorprenderse si evitan cumplir el modelo de RSC. Lo interesante de regular este modelo es que finalmente propone prevenir y eliminar los efectos colaterales, lo que obviamente implica ser más proactivo; pero a su vez, implica la posibilidad de limitar a la empresa al uso de nuevas tecnologías o estrategias -por pensar

4. En Gran Bretaña las alianzas público-privadas han permitido invertir en proyectos de bienestar social (educación, transporte, salud, prisiones). Se encuentran este tipo de alianzas en otros países europeos como España, Francia y Alemania, aunque cabe resaltar que la mayor inversión que se realiza a partir de alianzas público-privadas es en autopistas, agua potable, eliminación de residuos, educación y salud.

5. Como es el caso de Nike, empresa multimillonaria acusada de utilizar a niños de 11 años para la fabricación de su calzado de baloncesto Air Jordan (valorado en 125 dólares el par en EE. UU.) con una paga de 11 centavos por hora. Otro ejemplo es el caso de PetroBras, que es reconocida como una de las empresas que mayor cantidad de gases de carbono emiten, contaminando así el medioambiente y contribuyendo al cambio climático. 
en posibles efectos- que además aumentaría los costos y pondría en riesgo el posicionamiento de la empresa en el mercado por verse superado por su competencia.

La relación entre el voluntariado y la obligación debería mantenerse equilibrada de manera que existan normativas básicas en lo que respecta a la RSC que sean impuestas por organizaciones estatales y que a su vez exista un modelo ético voluntario, logrando que la efectividad de las actividades de esta índole, funcionen correctamente por la dependencia que tiene este modelo a una fuerza institucional (Vera y Hernández 2017). Siendo así, se consolidan niveles mínimos de bienestar social y transparencia empresarial en actividades y toma de decisiones, lo que implica que el modelo de RSC implementado de manera voluntaria por las empresas no generen grandes impactos en sus stakeholders; pero que, junto a regulaciones estatales, se impulsa el desarrollo social en aspectos más grandes. En otras palabras, la regulación se complementa al modelo de RSC en las organizaciones ya que facilitan el alcance del desarrollo sostenible aplicado a la sociedad en general.

\section{Conclusiones}

Es necesario tener en cuenta el gran sistema del que forma parte una compañía -micro y macroentorno- porque, en efecto, el desarrollo, el crecimiento, las actividades, los proyectos, entre otros, de una compañía, dependen de este sistema, lo que implica que se generen a su vez importantes impactos en diferentes áreas, social, ambiental, económica y política, convirtiéndolas también en actores involucrados involuntariamente. Por este motivo, la teoría de sistemas y la RSC se deben estudiar y aplicar de manera complementaria en la realidad; sin embargo, el definir de qué manera se debe manejar la teoría de sistemas crea dos opciones: como un sistema abierto, como menciona Bertalanffy, que conllevaría a la internalización de las externalidades, o un sistema cerrado como lo menciona Luhmann, que conduce a la necesidad de la tipificación legal de la RSC.

Al final, el modelo de RSC genera una gran controversia con la escuela clásica de la administración -pensamiento capitalista empresarial-por lo que es necesario la intervención estatal a través de la correspondiente legislación 
que regule las actividades cotidianas de la empresa, mitigando los posibles efectos en terceros y que, a su vez, promueva el bienestar colectivo. Es decir, se debe definir una normativa en base al modelo de responsabilidad social que no reduzca las capacidades de incrementar la rentabilidad de las empresas, pero que sí defina límites para el desarrollo de todas las actividades económicas. De esta manera el centro de cada actividad son los stakeholders que promueven el desarrollo sostenible empresarial, pues las organizaciones no pueden existir sin entorno. En realidad, la implementación efectiva de la RSC se convierte en una gran aspiración: llegar lo más lejos posible, maximizando la capacidad de operación de la empresa, pero siempre tomando en cuenta como centro de toda actividad empresarial el bienestar colectivo. Además, como es difícil definir el nivel de sensibilidad o voluntariedad que tiene una compañía hacia su entorno, se deben establecer regulaciones estatales que condicionen su comportamiento (lo que implica un modelo político para cada industria de la economía, pues como es lógico cada actividad tiene efectos diferentes que se podrían mitigar o eliminar por completo).

A pesar de que el pensamiento empresarial se enfoca en que los objetivos sociales y económicos son incompatibles, el comportamiento de las empresas debe dirigirse a la generación de rentabilidad, guiándose por normas y leyes estatales, definidas en base al bienestar social, y que tengan como fin el desarrollo sostenible. Sin embargo, no se puede dejar de lado que la RSC es un modelo de voluntariedad de cada compañía. En otras palabras, las empresas deben generar beneficios propios y para su entorno, invirtiendo capital y tiempo de la mano de las regulaciones estatales en conjunto con modelos voluntarios, por lo que los diferentes programas de RSC deberían ser vistos como un modelo o estrategia con la cual lo único que generaría es ventajas competitivas. Por esto, es necesario juntar la teoría de sistemas clásica, que ve a la empresa como un sistema abierto, y la teoría de sistemas contemporánea, que define a la empresa como un sistema limitado a los componentes internos de la misma, de manera que exista obligatoriedad en ciertos temas de responsabilidad social corporativa y diferentes modelos que se puedan implementar en las diferentes industrias de forma voluntaria, teniendo en cuenta la ventaja competitiva que se genera a corto plazo y la sostenibilidad a largo plazo. 


\section{Referencias}

Agudelo, Christian. 2013. "Sostenibilidad como modelo de crecimiento". Diario La República. Perú, 25 de abril de 2013. Consulta: abril de 2013. 〈https://issuu.com/diario_larepubli ca/docs/esp_15_casos_exitos_rse_abril_25_de_2013).

Carbon Trust. 2018. Energy Efficiency Programme Design \& Delivery Carbon Trust. Consulta: septiembre de 2018. 〈https://www.carbontrust.com/client-services/programmes/ programmes/>.

Carroll, Archie, y Kareem Shabana. 2010. "The Business Case for Corporate Social Responsibility: A Review of Concepts, Research and Practice”. International Journal of Management Reviews 12, No. 1: 85-105.

De la Cuesta, Marta, y Carmen Valor. 2003. "Responsabilidad social de la empresa: concepto, medición y desarrollo en España”. Boletín económico del ICE, No. 2755: 7-20.

Fernández, José Luis. 2016. De la RSC a la sostenibilidad corporativa: una evolución necesaria para la creación de valor. Consulta: abril de 2018. 〈https://www.researchgate.net/ publication/297757785_De_la_RSC_a_la_sostenibilidad_corporativa_una_evolucion_ necesaria_para_la_creacion_de_valor».

Fleming, Peter, John Roberts y Christina Garsten. 2013. "In Search of Corporate Social Responsibility: Introduction to Special Issue”. Organization 20, No. 3: 337-348.

Global Reporting Initiative. s. f. About GRI. Consulta: septiembre de 2018. «ttps://www. globalreporting.org/Information/about-gri/Pages/default.aspx>.

Godwin, Neva. 2007. "Internalizando externalidades: haciendo que los mercados y las sociedades funcionen mejor". Opinion Sur, No. 52: 1-6.

Golob, Urša, Trine Susanne Johansen, Anne Ellerup Nielsen y Klement Podnar. 2014. "Corporate Social Responsibility as a Messy Problem: Linking Systems and Sensemaking Perspectives". Systemic Practice and Action Research 27, No. 4: 363-376.

Gutiérrez, Roberto, y Laura González. 2011. "Normas internacionales y políticas públicas". En Antonio Vives y Estrella Peinado, edits., La responsabilidad social de la empresa en América Latina, 126-141. Nueva York: Banco Interamericano de Desarrollo.

Industrias Lácteos. 2018. "Valor compartido-TONICORP”. 2018. Consulta: febrero de 2018. 〈http://www.tonicorp.com/valor-compartido.html〉.

ISO. s. f. "Standards". Consulta: mayo de 2018. 〈https://www.iso.org/standards.html〉.

Jaramillo, Juan Guillermo. 2007. "Evolución histórica de los conceptos de responsabilidad social empresarial y balance social”. Semestre Económico 10, No. 20: 87-102.

Kamppinen, Matti, Petteri Vihervaara y Nina Aarras. 2008. "Corporate Responsibility and Systems Thinking: Tools for Balanced Risk Management”. International Journal of Sustainable Society 1, No. 2: 158-171.

Luhmann, Niklas, y Darío Rodríguez Mansilla. 1997. Organización y decisión. Barcelona: Anthropos. 
Marín Rives, Longinos, y Alicia Rubio Bañón. 2008. “¿Moda o factor competitivo? Un estudio empírico de responsabilidad social corporativa en pyme". Tribuna de Economía ICE, No. 842: 177-193.

Mascareño, Aldo, y Camilo Drago. 2016. "Nothing More than Feelings? De la compasión individual a la compasión sistémica en las organizaciones modernas". Economía y Política 3, No. 2: 85-117.

Porter, Michael, y Mark Kramer. 2006. "Estrategia y sociedad”. Harvard Business School Review 84, No. 12: 42-56.

Sabogal, Javier. 2008. “Aproximación y cuestionamientos al concepto responsabilidad social empresarial”. Revista Facultad de Ciencias Económicas XVI, No. 1: 179-195.

Teubner, Gunther. 2012. Constitutional Fragments: Societal Constitutionalism and Globalization. Oxford: OUP.

TOMS. 2006. "Improving Lives | TOMS® Giving”. Consulta: mayo de 2018. «ttps://www. toms.com/improving-lives〉.

Toro, Daniela. 2006. "Enfoque estratégico de la responsabilidad social corporativa: revisión de la literatura académica". Intangible Capital 2, No. 4: 338-358.

Valencia, Pilar, Manuela Escobar y Felipe Calderón. 2017. "Una mirada alternativa a la responsabilidad social empresarial desde el control de constitucionalidad en una economía emergente en pos conflicto". Revista Eletrônica do Curso de Direito da UFSM 12, No. 2: 692-708.

Valentinov, Vladislav. 2013. "Corporate Social Responsibility and Sustainability: Insights from Boulding and Luhmann”. International Journal of Sustainable Development \& World Ecology 20, No. 4: 317-324.

Vera, José, y Mabel Hernández. 2017. "La responsabilidad social empresarial desde la teoría sociopoiética”. En Martha Santa Ana y Esther Morales, coords., La responsabilidad social de las organizaciones: diversidad y complejidad organizacional en América Latina, 212245. Ciudad de México: Hess.

Von Bertalanffy, Ludwig. 1976a. Teoria general de los sistemas: fundamentos, desarrollo, aplicaciones. Ciudad de México: Fondo de Cultura Económica.

---. 1976b. General System Theory: Foundations, Development, Applications. Nueva York: George Brazillier. 